\title{
Efficacy of Gamma Knife Radiosurgery for Recurrent High-Grade Gliomas with Limited Tumor Volume
}

\author{
Young-Jun Cheon, M.D., Tae-Young Jung, M.D., Ph.D., Shin Jung, M.D., Ph.D., In-Young Kim, M.D., Ph.D., \\ Kyung-Sub Moon, M.D., Ph.D., Sa-Hoe Lim, Ph.D. \\ Department of Neurosurgery, Chonnam National University Hwasun Hospital, Chonnam National University School of Medicine, Hwasun, \\ Korea
}

Objective : This study aims to determine whether gamma knife radiosurgery (GKR) improves survival in patients with recurrent highgrade gliomas.

Methods : Twenty nine patients with recurrent high-grade glioma underwent 38 GKR. The male-to-female ratio was 10 : 19 , and the median age was 53.8 years (range, 20-75). GKR was performed in 11 cases of recurrent anaplastic oligodendrogliomas, five anaplastic astrocytomas, and 22 glioblastomas. The median prescription dose was 16 Gy (range, 10-24), and the median target volume was $7.0 \mathrm{~mL}$ (range, 1.1-15.7). Of the 29 patients, 13 (44.8\%) received concurrent chemotherapy. We retrospectively analyzed the progression-free survival (PFS) and overall survival (OS) after GKR depending on the Eastern Cooperative Oncology Group (ECOG) performance status (PS), pathology, concurrent chemotherapy, radiation dose, and target tumor volume.

Results : Starting from when the patients underwent GKR, the median PFS and OS were 5.0 months (range, 1.1-28.1) and 13.0 months (range, 1.1-75.1), respectively. On univariate analysis, the median PFS was significantly long in patients with anaplastic oligodendroglioma, ECOG PS 1, and target tumor volume less than $10 \mathrm{~mL}(p<0.05)$. Meanwhile, on multivariate analysis, patients with ECOG PS 1 and target tumor volume less than $10 \mathrm{~mL}$ showed improved PFS ( $p=0.043$ and $p=0.007$, respectively). The median OS was significantly increased in patients with ECOG PS 1 and tumor volume less than $10 \mathrm{~mL}$ on univariate and multivariate analyses $(p<0.05)$.

Conclusion : GKR could be an additional treatment option in recurrent high-grade glioma, particularly in patients with good PS and limited tumor volume.

Key Words : Recurrence · Glioma · Gamma knife radiosurgery · Tumor volume.

\section{INTRODUCTION}

Current treatment modalities for high-grade gliomas include surgery, adjuvant radiotherapy, and maintenance chemother- apy. Many patients are treated with systemic chemotherapy as an initial and salvage management approach in the form of bevacizumab, temozolomide, irinotecan, and nitrosoureas ${ }^{13,18,19)}$. Despite recent advances in radiation, chemotherapy, and surgi-

- Received : September 28, 2017 •Revised : December 26, 2017 •Accepted : January 1, 2018

- Address for reprints : Tae-Young Jung, M.D., Ph.D.

Department of Neurosurgery, Chonnam National University Hwasun Hospital, 322 Seoyang-ro, Hwasun-eup, Hwasun 58128, Korea

Tel : +82-61-379-7666, Fax : +82-61-379-7373, E-mail : jung-ty@chonnam.ac.kr

This is an Open Access article distributed under the terms of the Creative Commons Attribution Non-Commercial License (http://creativecommons.org/licenses/by-nc/4.0) which permits unrestricted non-commercial use, distribution, and reproduction in any medium, provided the original work is properly cited. 
cal care of patients with high-grade gliomas, treatment of these neoplasms remains challenging for neurosurgeons and oncologists $^{5}$. Recurrence of high-grade glioma is common owing to limited treatment options. Currently, salvage treatment options for recurrent high-grade glioma include re-resection, re-irradiation including stereotactic radiosurgery or fractionated radiotherapy, single/poly-chemotherapy, and targeted drug delivery $^{13)}$. Despite the higher risk of distant brain lesions or subependymal spread, almost all patients develop tumor recurrence within or adjacent to the primary tumor bed ${ }^{10)}$. Radiation therapy is among the primary treatment modalities for high-grade glioma. Meanwhile, re-irradiation for local recurrence is rarely considered because of the possibility of radiation-induced injury ${ }^{2,6)}$.

Even though gliomas are infiltrative with ill-defined margins, stereotactic radiosurgery has been reported to be an effective treatment modality ${ }^{1,4,7,10,17)}$. It can deliver high-dose radiation on the recurred tumor bed while sparing adjacent normal brain tissues. Stereotactic surgery has been reported to improve survival in young patients with less tumor volume and low histologic grade and oligodendroglial pathology ${ }^{8,10}$. Meanwhile, gamma knife radiosurgery (GKR) may improve survival in patients with small treatment volume. In this study, we retrospectively investigated the clinical outcome of recurrent high-grade gliomas after GKR.

\section{MATERIALS AND METHODS}

\section{Clinical characteristics of patients with recurrent high-grade glioma}

From April 2004 to December 2016, 29 patients with recurrent high-grade glioma underwent 38 cases of GKR. GKR was done with type $\mathrm{C}$ with/without an automatic positioning system. The male-to-female ratio was $10: 19$, and the median age was 53.8 years (range, 20-75). GKR was performed for 11 cases of pathologically diagnosed anaplastic oligodendrogliomas, five anaplastic astrocytomas, and 22 glioblastomas. The tumors were graded following the World Health Organization classification ${ }^{15)}$. The tumor recurrence was diagnosed with contrast-enhanced brain magnetic resonance (MR) images including diffusion, MR spectroscopy and perfusion images ${ }^{11)}$.C-methionine (MET) positron emission tomography (PET) images were checked to differentiate the recurred lesion from radiation necrosis, and delineate the target volume for GKR. Before GKR, 29 patients underwent treatments as follows : radiotherapy of 59.4 Gy plus concomitant and adjuvant temozolomide $(\mathrm{n}=12)$, radiotherapy of 40-59.4 Gy $(\mathrm{n}=17)$, temozolomide chemotherapy $(\mathrm{n}=6)$, procarbazine, lomustine, and vincristine (PCV) chemotherapy $(n=4)$, nimustine and cisplatin $(n=2)$, carmustine and cisplatin $(\mathrm{n}=1)$, and bevacizumab and irinotecan $(\mathrm{n}=1)$. The Eastern Cooperative Oncology Group (ECOG) performance status (PS) scales (grades 0-5) were used to assess disease progression and how the disease and treatments affected activities of daily living to determine patient prognosis and appropriate treatment ${ }^{14)}$. An ECOG PS grade of 0 indicates that the patient is fully active and able to perform all predisease activities without restriction. A patient with an ECOG grade 1 PS is restricted in physically strenuous activity but is ambulatory and able to perform light or sedentary work, for example, light housework or office work). Grade 2 indicates that a patient is ambulatory and capable of all self-care but is unable to perform any work activities for more than $50 \%$ of

Table 1. Characteristics of 29 patients with 38 GKR cases of recurrent high-grade gliomas

\begin{tabular}{lc}
\hline Patient characteristic & Value \\
\hline Patients & $10 / 19$ \\
Male/female & 53.8 (range, 20-75) \\
Median age (years) & 5 \\
Pathology & 11 \\
Anaplastic astrocytomas & 22 \\
Anaplastic oligodendrogliomas & \\
Glioblastomas & 26 \\
ECOG PS & 10 \\
1 & 2 \\
2 & \\
3 & 16 (range, 10-24) \\
Characteristics of GKR & 7.0 (range, 1.1-15.7) \\
Median radiation dose (Gy) & \\
Median target volume (mL) & 9 \\
Chemotherapy combined & 3 \\
Temozolomide & 1 \\
PCV & \\
ICE & \\
\hline GKR : gamma knife radiosurgery, ECOG : the Eastern Cooperative Oncology \\
Group, PS : performance status, PCV : procarbazine, CCNU, and vincristine, \\
ICE : ifosfamide, carboplatin and etoposide \\
\hline
\end{tabular}


waking hours. Grade 3 indicates that a patient is capable of only limited self-care and is confined to a bed or a chair for more than $50 \%$ of waking hours. When GKR was performed, 26 cases had an ECOG PS grade of 1, 10 had PS grade 2, and two had PS grade 3 . The clinical characteristics are summarized in Table 1.

\section{Gamma knife radiosurgery for recurrent high- grade gliomas}

GKR was performed using Leksell Gamma Plan ${ }^{\circledR}$ (Elekta Instrument AB, Stockholm, Sweden) radiosurgery planning software. The median prescription dose was 16 Gy (range, 10-24), and the median target volume was $7.0 \mathrm{~mL}$ (range, 1.115.7). After GKR, the median follow-up duration was 11.5 months (range, 1.1-75.1). GKR was targeted on relatively wellcircumscribed recurred lesions on brain MR images and/or MET-PET images. Most target lesions were MR-enhanced, and MET-PET images was used to delineate the target lesion with/without contrast enhancement. A total of 13 of the 29 (44.8\%) patients received concurrent chemotherapy consisting of temozolomide ( $n=9)$; PCV $(n=3)$; and ifosfamide, carbopla- tin, and etoposide (ICE) $(n=1)$.

After GKR with/without chemotherapy, the patterns of recurrence were categorized into local, distant, and cerebrospinal fluid (CSF) dissemination. Local recurrence was defined as recurrence at the primary tumor site, while distant recurrence was defined as a spatially separated recurrence within the brain. CSF dissemination was defined as recurrence at the ventricles, subarachnoid space, and spine as observed from imaging studies. The recurrence occurred in 29 cases out of 38. Local recurrence was noted in 14 patients. Of them, 10 underwent further treatment as follows : operation $(n=3)$, temozolomide chemotherapy $(n=4)$, PCV chemotherapy $(n=2)$, and bevacizumab and irinotecan $(n=1)$. Distant recurrence developed in 12 patients, including two combined with local recurrence. Of the 12 patients, 10 underwent further treatment : GKR $(n=6)$, temozolomide $(n=1), \operatorname{PCV}(n=1)$, and nimustine and cisplatin $(n=2)$. Meanwhile, CSF dissemination was noted in three patients. Of them, two were subjected to additional treatment: radiotherapy and ICE chemotherapy $(n=1)$ and nexavar chemotherapy $(\mathrm{n}=1)$.

Table 2. Univariate analysis related with PFS and OS

\begin{tabular}{|c|c|c|c|c|}
\hline Variable & Median PFS (range, months) & $p$-value & Median OS (months) & $p$-value \\
\hline \multicolumn{5}{|l|}{ Pathology } \\
\hline Anaplastic oligodendrogliomas & $6.9(4.4-18.4)$ & 0.001 & $18.2(7.7-75.1)$ & 0.107 \\
\hline Anaplastic astrocytomas & $3.6(2.5-5.2)$ & 0.180 & $10.8(8.4-16.8)$ & 0.257 \\
\hline Glioblastomas & $4.5(1.1-28.1)$ & & $13.4(1.1-55.3)$ & \\
\hline \multicolumn{5}{|l|}{ ECOGPS } \\
\hline 1 & $6.5(2.6-28.1)$ & 0.024 & $16.8(4.2-75.1)$ & 0.000 \\
\hline 2 & $4.2(1.1-10.1)$ & 0.692 & $7.7(3.7-19.4)$ & 0.042 \\
\hline 3 & $1.1(1.1-4.5)$ & & $1.1(1.1-4.5)$ & \\
\hline \multicolumn{5}{|l|}{ GKS dose } \\
\hline$\geq 18 \mathrm{~Gy}$ & $4.9(1.1-25.9)$ & 0.928 & $14.1(1.1-75.1)$ & 0.801 \\
\hline$<18 \mathrm{~Gy}$ & $5.2(1.1-28.1)$ & & $11.3(3.7-55.3)$ & \\
\hline \multicolumn{5}{|l|}{ Tumor volume } \\
\hline$\leq 10 \mathrm{~mL}$ & $5.8(1.6-28.1)$ & 0.008 & $14.7(3.7-75.1)$ & 0.002 \\
\hline$>10 \mathrm{~mL}$ & $3.4(1.1-7.7)$ & & $8.4(1.1-13.0)$ & \\
\hline \multicolumn{5}{|l|}{ Chemotherapy combined } \\
\hline No chemotherapy & $5.2(1.1-25.9)$ & 0.740 & $11.6(1.1-75.1)$ & 0.945 \\
\hline Chemotherapy & $4.5(1.6-28.1)$ & & $14.7(3.7-28.1)$ & \\
\hline
\end{tabular}

PFS : progression-free survival, OS : overall survival, ECOG : the Eastern Cooperative Oncology Group, PS : performance status, GKS : gamma knife radiosurgery 

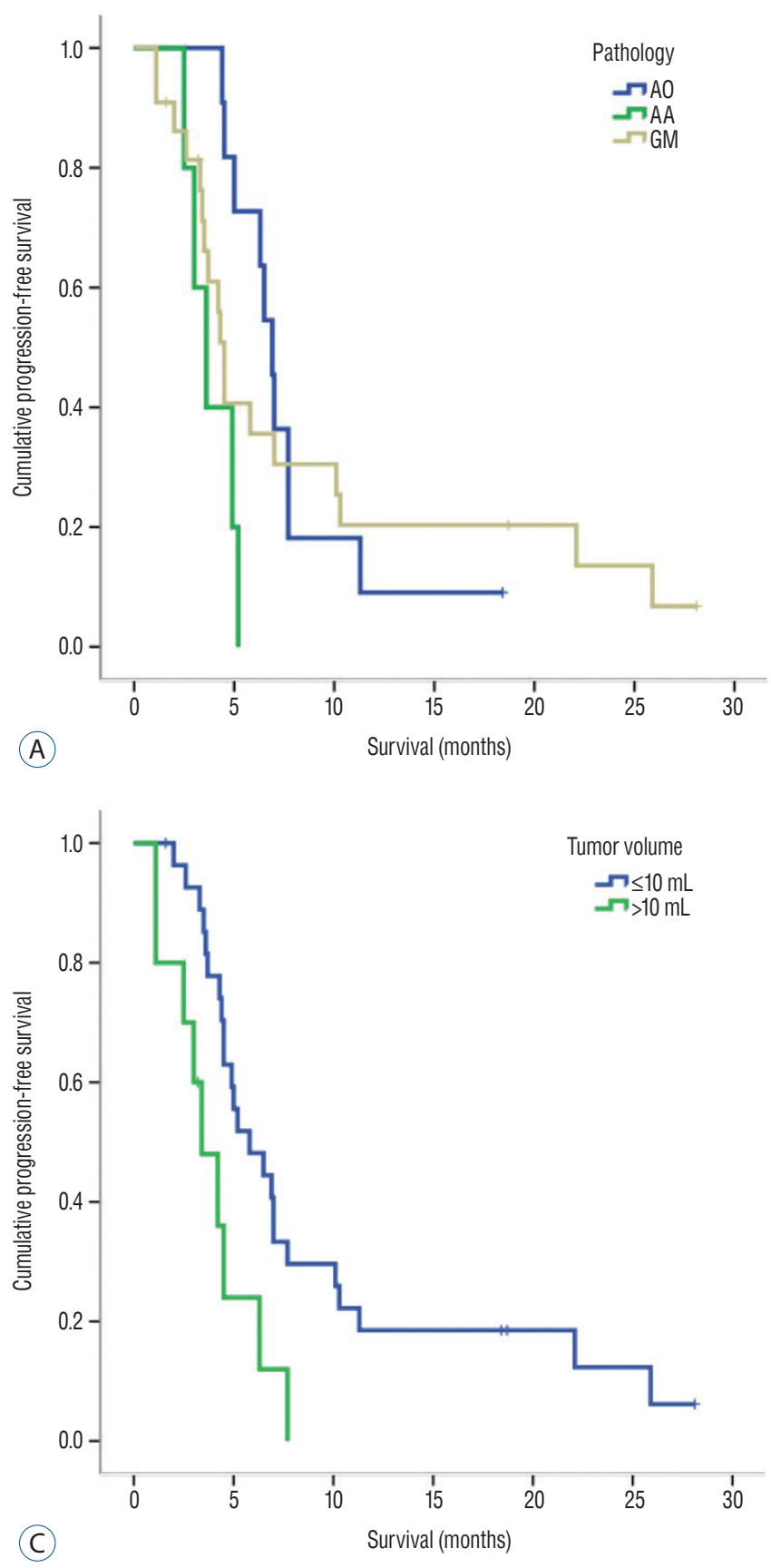

\section{Statistical analysis}

The median range of survival was defined as survival up to the last follow-up. Variables affecting progression-free survival (PFS) and overall survival (OS) after GKR were determined via univariate and multivariate analyses. The single variables were ECOG PS, pathology, concurrent chemotherapy, radiation dose, and target tumor volume. PFS was calculated from the date of surgery to the date of radiological progression or last

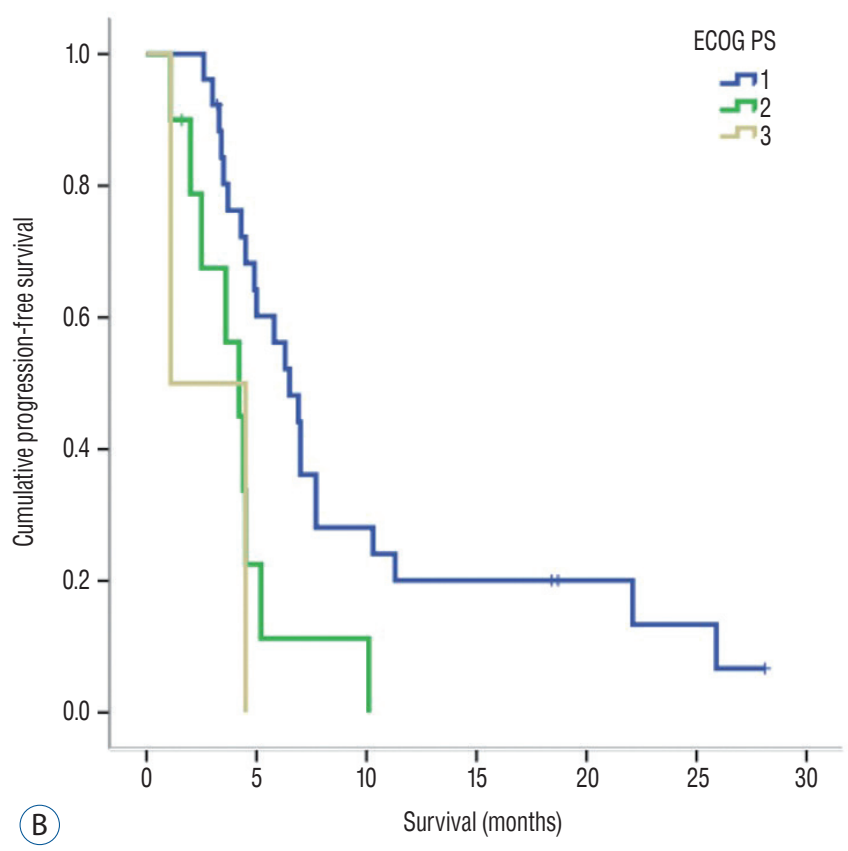

Fig. 1. Kaplan-Meier curve related to progression-free survival. A : Median PFS was significantly longer in patients with anaplastic oligodendroglioma than in those with glioblastoma ( $p=0.001)$. B : Survival of patients with ECOG PS 1 was statistically significantly different compared to those with ECOG PS $3(p=0.024)$. $\mathrm{C}$ : Survival of patients with tumor volume less than $10 \mathrm{~mL}$ was statistically significantly different compared to those with tumor volume over $10 \mathrm{~mL}(p=0.008)$. $\mathrm{AO}$ : anaplastic oligodendroglioma, AA : anaplastic astrocytoma, GM : glioblastoma; PFS : progression-free survival, ECOG : the Eastern Cooperative Oncology Group, PS: performance status.

follow-up visit, and OS was calculated from the date of surgery to the date of death or last follow-up. Survival probability was calculated using the Kaplan-Meier method, and comparisons were performed with the log-rank test. We examined variables using the Cox proportional hazard analysis model to identify the independent predictors of survival. All statistical analyses were performed with a significance level of $p<0.05$ using SPSS version 21.0 (SPSS, Chicago, IL, USA). 

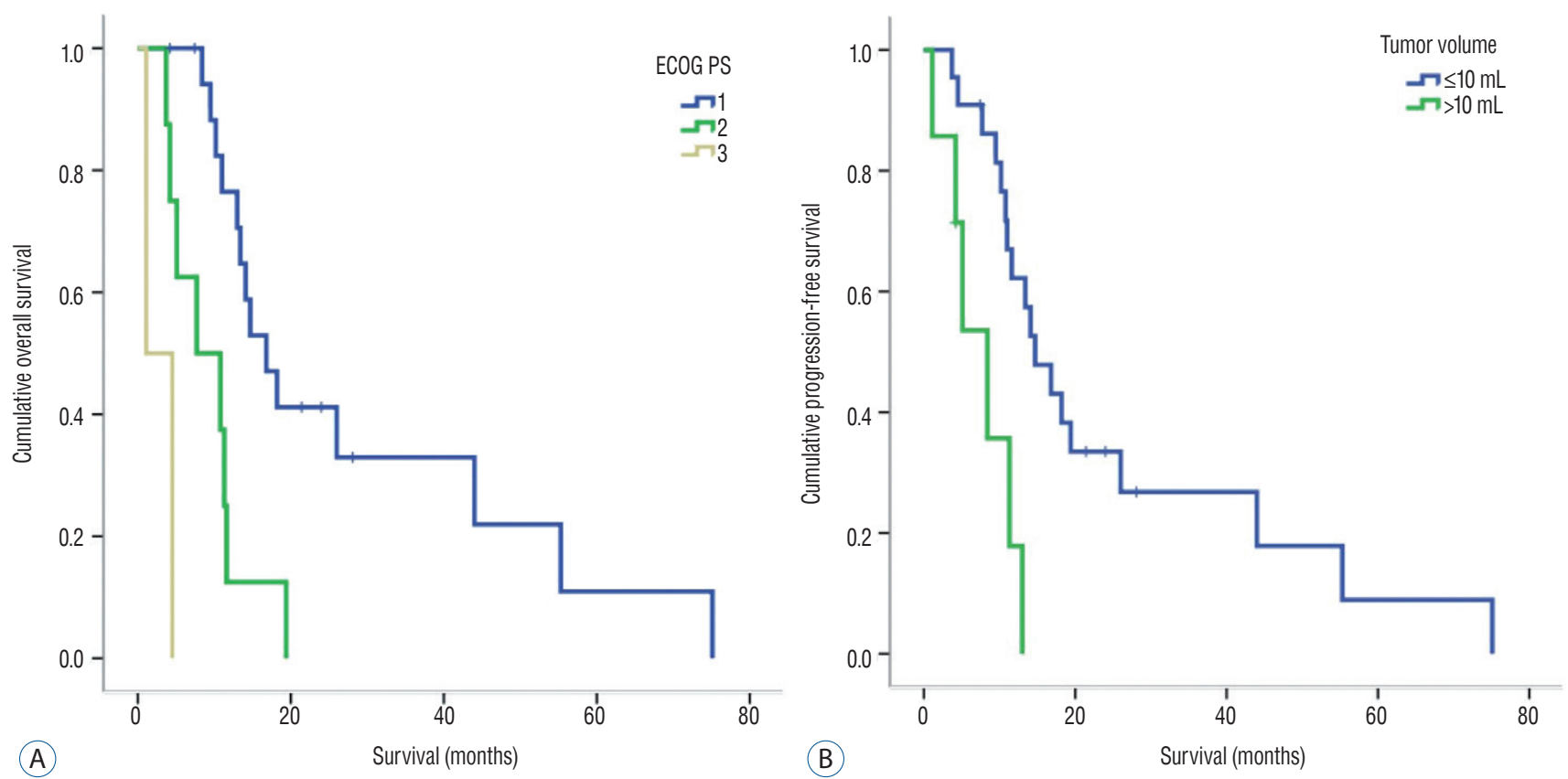

Fig. 2. A : Kaplan-Meier curve related with overall survival. Patients with ECOG PS 1 and 2 had better OS compared to those with ECOG PS 3 ( $p=0.000$ and 0.042 , respectively). B : Patients with tumor volume less than $10 \mathrm{~mL}$ had better OS compared to those with tumor volume over $10 \mathrm{~mL}(p=0.002)$. ECOG : the Eastern Cooperative Oncology Group, PS : performance status, OS : overall survival.

Table 3. Multivariate analysis related with PFS and OS

\begin{tabular}{lccc}
\hline Variable & $\begin{array}{c}\text { Hazard } \\
\text { ratio }\end{array}$ & $p$-value & $\mathbf{9 5 \%} \mathrm{Cl}$ \\
\hline PFS related & & & \\
$\quad$ Pathology & & & \\
$\quad$ Anaplastic oligodendrogliomas & 0.658 & 0.372 & $0.262-1.649$ \\
$\quad$ Anaplastic astrocytomas & 2.489 & 0.122 & $0.784-7.895$ \\
$\quad$ Tumor volume & & & \\
$\quad \leq 10 \mathrm{~mL}$ & 0.273 & 0.007 & $0.106-0.703$ \\
$\quad$ ECOG PS & & & \\
$\quad 1$ & 0.190 & 0.043 & $4.710-8.290$ \\
2 & 0.366 & 0.238 & $2.465-5.935$ \\
OS related & & & \\
Tumor volume & & & \\
$\quad \leq 10 \mathrm{~mL}$ & 0.239 & 0.015 & $0.075-0.761$ \\
ECOG PS & & & \\
1 & 0.018 & 0.000 & $0.002-0.161$ \\
2 & 0.062 & 0.012 & $0.007-0.541$ \\
\hline
\end{tabular}

References: glioblastoma, tumor volume $>10 \mathrm{~mL}$, ECOG PS 3. PFS : progression-free survival, OS : overall survival, $\mathrm{Cl}$ : confidence interval, ECOG : the Eastern Cooperative Oncology Group, PS : performance status

\section{RESULTS}

\section{Prognostic factors related with progression-free survival and overall survival}

Starting from when the patients underwent GKR, the median PFS and OS were 5.0 months (range, 1.1-28.1) and 13.0 months (range, 1.1-75.1), respectively. The prognostic factors related to PFS and OS on univariate analysis are summarized in Table 2. The median PFS was significantly longer in patients with anaplastic oligodendroglioma (6.9 months [range, 4.4-18.4]) compared to those with glioblastoma (4.5 months [range, 1.1-28.1]) $(p=0.001)$ (Fig. 1A). Moreover, survival of patients with an ECOG PS 1 and tumor volume less than $10 \mathrm{~mL}$ was statistically significantly different compared to those with an ECOG PS 3 and tumor volume over $10 \mathrm{~mL}$ ( $p=0.024$ and 0.008 , respectively). The median PFS of those with an ECOG PS 1 and PS 3 was 6.5 months (range, 2.6-28.1) and 1.1 months (range, 1.1-4.5), respectively (Fig. 1B). Meanwhile, the median PFS of patients with a tumor volume less than $10 \mathrm{~mL}$ was 5.8 months (range, 1.6-28.1), while for those with over $10 \mathrm{~mL}$ was 3.4 months (range, 1.1-7.7) (Fig. 1C). Differences in PFS based on radiation dose and combined chemotherapy were not statistically significant. Meanwhile, differences in OS in terms of ECOG PS 

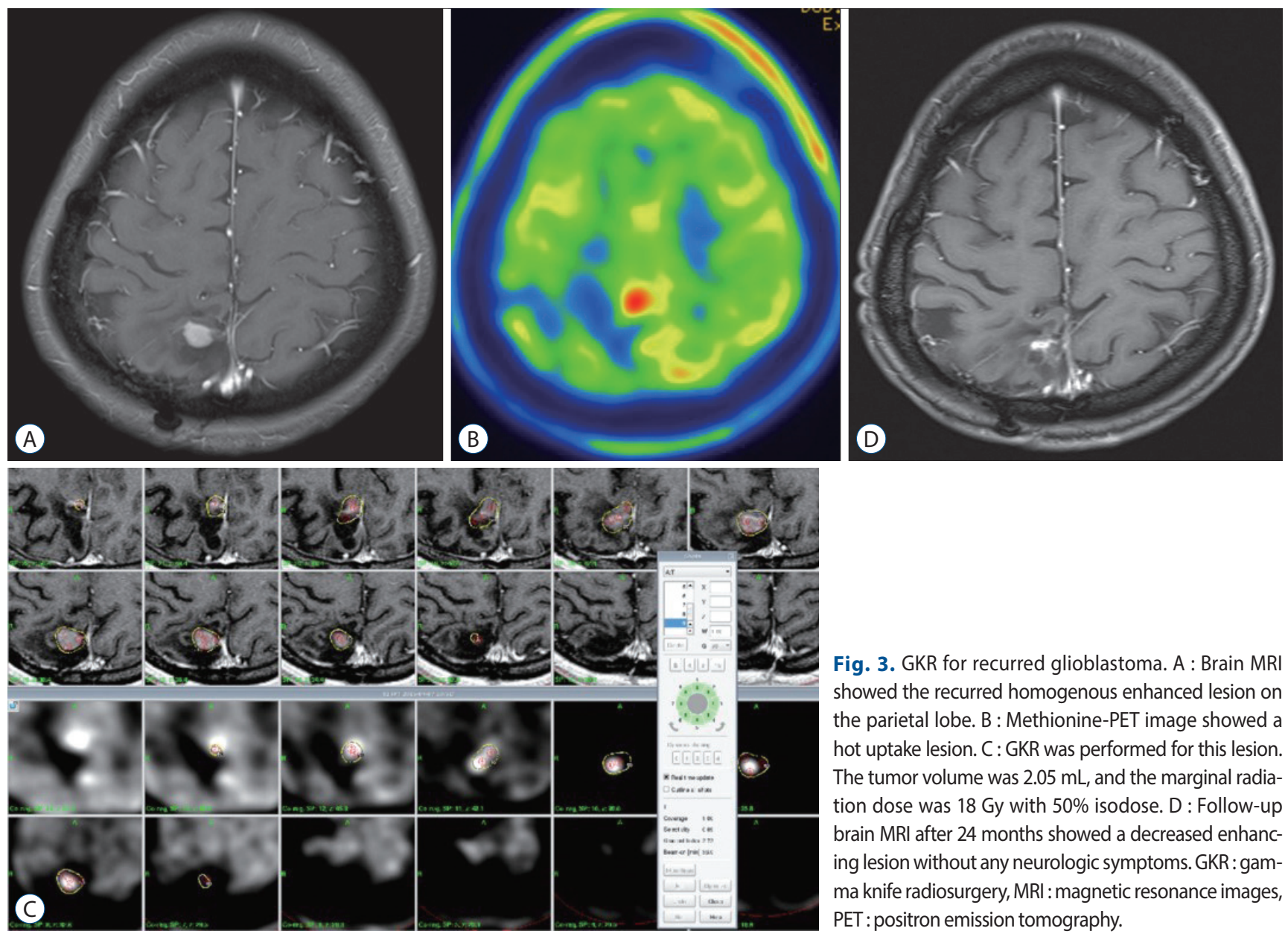

Fig. 3. GKR for recurred glioblastoma. A : Brain MRI showed the recurred homogenous enhanced lesion on the parietal lobe. B : Methionine-PET image showed a hot uptake lesion. C: GKR was performed for this lesion. The tumor volume was $2.05 \mathrm{~mL}$, and the marginal radiation dose was 18 Gy with $50 \%$ isodose. D : Follow-up brain MRI after 24 months showed a decreased enhancing lesion without any neurologic symptoms. GKR: gamma knife radiosurgery, $\mathrm{MRI}$ : magnetic resonance images, PET : positron emission tomography.

and tumor volume were statistically significant $(p=0.000$ and 0.002 , respectively). The median OS of patients with an ECOG PS 1, 2, and 3 was 16.8 months (range, 4.2-75.2), 7.7 months (range, 3.7-19.4), and 1.1 months (range, 1.1-4.5), respectively (Fig. 2A). The median OS of patients with tumor volume less than $10 \mathrm{~mL}$ was 14.7 months (range, 3.7-75.1), while for those with over $10 \mathrm{~mL}$ was 8.4 months (range, 1.1-13.0) (Fig. 2B).

Factors related to PFS and OS on multivariate analysis are listed in Table 3. Patients with tumor volume less than $10 \mathrm{~mL}$ had longer PFS (hazard ratio [HR] 0.273, 95\% confidence interval [CI] 0.106-0.703, $p=0.007$ ) and OS (HR 0.239, 95\% CI $0.075-0.761, p=0.015)$ than those with over $10 \mathrm{~mL}$. Moreover, patients with ECOG PS 1 showed longer PFS (HR 0.190, 95\% CI 4.710-8.290, $p=0.043$ ) than those with an ECOG PS 3. Further, patients with ECOG PS 1 and 2 (HR 0.018, 95\% CI 0.002$0.161, p=0.000$ ) had longer OS than those with ECOG PS 3 (HR $0.062,95 \%$ CI $0.007-0.541, p=0.012$ ).

\section{Case presentation 1 : GKR for recurred glioblastoma}

We present a case of a 21-year-old woman noted to have newly developed lesions on the right parietal lobe on routine follow-up radiological imaging. Seven years ago, she underwent craniotomy and tumor resection. Pathologically, glioblastoma was diagnosed. She received concomitant radiotherapy of 59.4 Gy and 6 cycles of adjuvant chemotherapy using temozolomide. Brain magnetic resonance images (MRI) showed a homogenous enhanced lesion on the parietal lobe, and methionine-PET image showed a hot uptake lesion (Fig. 3A and B). GKR was performed for this lesion. The tumor volume was $2.05 \mathrm{~mL}$, and the marginal radiation dose was 18 Gy with 50\% isodose (Fig. 3C). A combined 12 cycles of temozolomide chemotherapy was then administered after GKR. Follow-up brain MRI 24 months after treatment showed decreased enhancing lesion without any neurologic symptoms (Fig. 3D). 

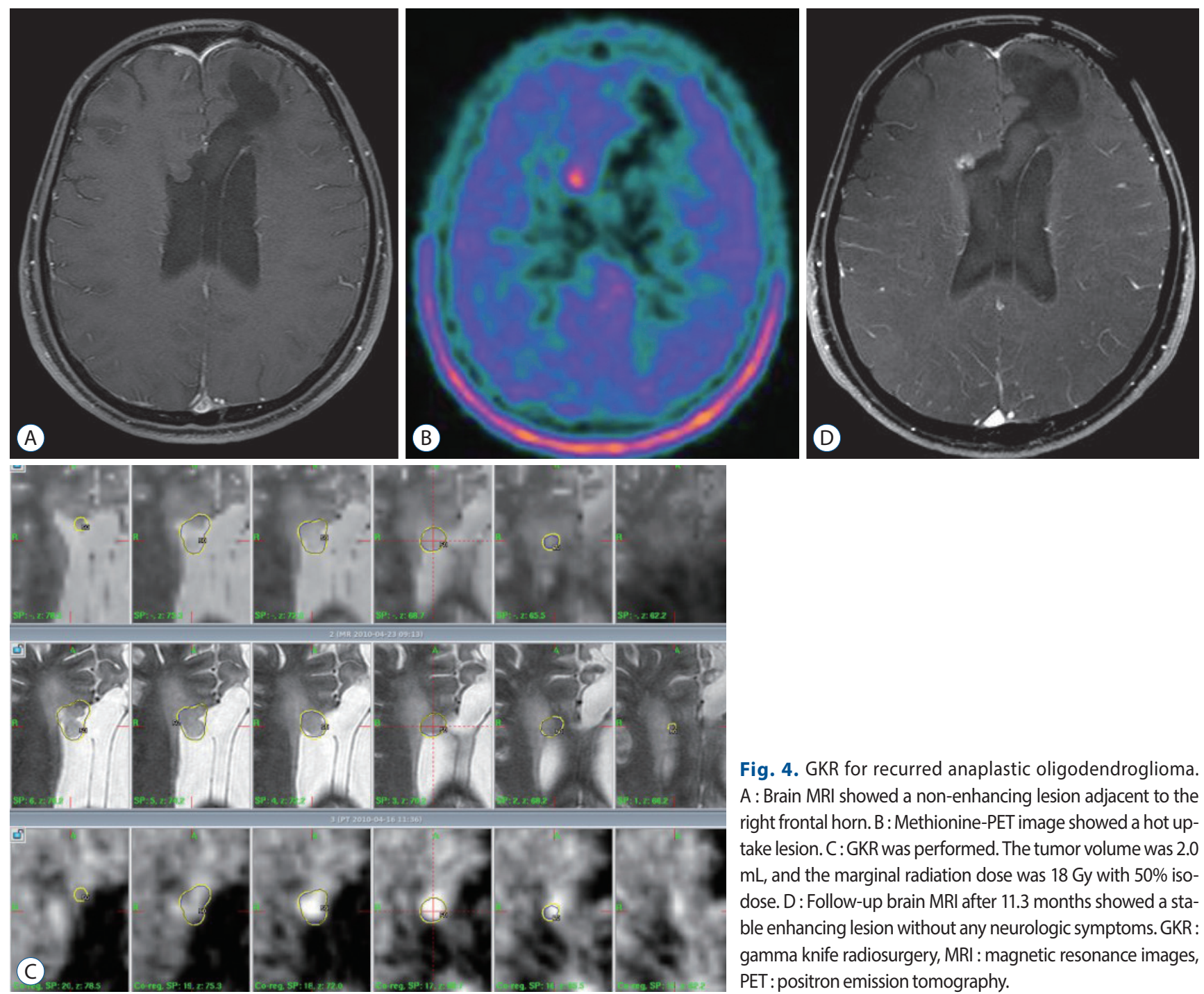

Fig. 4. GKR for recurred anaplastic oligodendroglioma. $A$ : Brain MRI showed a non-enhancing lesion adjacent to the right frontal horn. B: Methionine-PET image showed a hot uptake lesion. C: GKR was performed. The tumor volume was 2.0 $\mathrm{mL}$, and the marginal radiation dose was 18 Gy with $50 \%$ isodose. D : Follow-up brain MRI after 11.3 months showed a stable enhancing lesion without any neurologic symptoms. GKR: gamma knife radiosurgery, MRI : magnetic resonance images, $\mathrm{PET}$ : positron emission tomography.

\section{Case presentation 2 : GKR for recurred anaplastic oligodendroglioma}

We present a case of a 22-year-old man who underwent craniotomy and tumor resection. Pathologically, anaplastic oligodendroglioma was diagnosed. He received radiotherapy at a dose of 50.4 Gy. Within 4 years from diagnosis, re-operation, PCV chemotherapy, and temozolomide chemotherapy were performed for repeated recurrent lesion. Brain MRI showed a non-enhancing lesion adjacent to the right frontal horn, and methionine-PET image showed a hot uptake lesion (Fig. 4A and B). Consequently, GKR was performed. The tumor volume was $2.0 \mathrm{~mL}$, and the marginal radiation dose was $18 \mathrm{~Gy}$ with $50 \%$ isodose (Fig. 4C). Follow-up brain MRI after 11.3 months showed a stable enhancing lesion without any neuro- logic symptoms (Fig. 4D).

\section{DISCUSSION}

Although radiation therapy is effective for treating highgrade gliomas, irradiation and re-irradiation for local brain recurrence has been rarely considered because of the risk of radiation injury to the normal brain. The interval between the first and second irradiation is recommended to be at least six months ${ }^{2,6)}$. Radiation can be delivered through two techniques : fractionated 3D-conformal radiotherapy and single/fractionated stereotactic radiosurgery. Stereotactic radiosurgery can deliver a steep-dose gradient with geometrical accuracy and high 
conformity. With this technique, high doses can be delivered to small target volumes while sparing the surrounding normal healthy tissue. Through re-irradiation at the edge of the recurrent tumor bed, the tumor can be treated while preserving the normal tissue. For recurrent tumors located at different sites along the tumor bed, radiosurgery can individually target them. However, a large treatment volume can also expose a large area of normal tissues owing to the detrimental effect of high radiation dose $^{2)}$. Moreover, high tumor volume can increase the risk of side effects after radiosurgery.

For patients with anaplastic gliomas and glioblastomas, stereotactic radiosurgery was reported to result to a median PFS of 8.6 and 4.6 months, respectively, after salvage treatment for recurrent malignant gliomas ${ }^{10)}$. Historical data showed a survival benefit of such treatment modality as salvage strategy. In this study, the median PFS was 5.0 months after GKR. Depending on the pathology, the median PFS of those with anaplastic oligodendroglioma, anaplastic astrocytoma, and glioblastoma was 6.9, 3.6, and 4.5 months, respectively. Less tumor volume, low histologic grade, good PS, and young age were the prognostic factors significantly related with improved survival ${ }^{1,8,10,11,16)}$. Radiosurgery has a limited role for large treatment volume. However, tumor volume less than $10 \mathrm{~mL}$ and low histologic grade were significantly related with improved survival ${ }^{10)}$. Larson et al. ${ }^{11)}$ reported a better outcome in patients who were younger $(<70)$, in good general condition (Karnofsky performance score $>60$ ), with smaller tumor diameter $(<5 \mathrm{~cm})$, and tumors located in unifocal, hemispheric, supratentorial, and subcortical areas. In this study, PS and tumor volume less than $10 \mathrm{~mL}$ were associated with a favorable prognosis after GKR. Stereotactic radiosurgery can also be an alternative to open surgery for surgically inaccessible recurrent small lesions. Moreover, stereotactic radiosurgery is more beneficial than surgery for recurrent high-grade glioma or glioblastoma because of the high risk of complications in the latter treatment strategy ${ }^{1)}$.

Because of infiltrative nature of glioma, some viable cells adjacent to target lesion cannot be treated by stereotactic radiosurgery. Therefore, combined treatment with chemotherapy and stereotactic radiotherapy has resulted in improved survival. Kim et al." reported that the OS, PFS, and 6-month PFS were 15.5 months, 6.0 months, and $48.8 \%$ after combined treatment with GKR and temozolomide chemotherapy for recurrent glioblastoma. The median treatment volume was $9.8 \mathrm{~mL}$ for the combination group and $11 \mathrm{~mL}$ for the GKR alone group. The median radiation dose was 15 Gy. The combination of GKR and temozolomide statistically resulted to superior survival benefit compared to GKR alone and temozolomide alone. Conti et al. ${ }^{3)}$ reported that cyberknife stereotactic radiosurgery in combination with temozolomide led to an increased survival time of 12 months and a 66.7\% 6-month PFS. Minniti et al. ${ }^{12)}$ reported that after stereotactic radiosurgery combined with temozolomide as a salvage treatment, OS and PFS increased by 9.7 and 5 months, respectively, and the 6-month PFS rate was $42 \%$. Meanwhile, radiosurgery combined with chemotherapy can have a radio-sensitizing or other synergistic effect in patients with high-grade glioma ${ }^{19)}$. In this study, the GKR combined with chemotherapy group did not show improved survival. Estimating the combination effect was difficult due to the small number of patients and the variety of chemotherapeutic regimens used.

In this study, patients with anaplastic oligodendroglioma showed longer PFS than those with anaplastic astrocytoma and glioblastoma. Radiosurgery has been reported to play a role in oligodendroglioma management ${ }^{8,16)}$. After GKR for oligodendroglioma, several factors, including low tumor grade and small tumor volume, were associated with improved $\mathrm{PFS}^{8)}$. Patients with anaplastic oligodendroglioma showed an OS of $88.9 \%$ and $19.6 \% 1$ and 5 years after GKR, respectively. One case report showed that multiple courses of stereotactic re-irradiation controlled repeated recurrence without significant toxicity for anaplastic oligodendroglioma ${ }^{7}$. Radiotherapy, which is an essential treatment for those with anaplastic oligodendroglioma, prolonged the survival of such patient group ${ }^{18}$. Stereotactic radiosurgery had the advantage of providing localized radiation and could be an additional treatment option for recurred anaplastic oligodendroglioma.

The limitations of our study include a small patient population, pathologic diagnosis without genotype information, and heterogeneity of treatment strategy. On recurrent high-grade glioma, it could be difficult to clearly differentiate the tumor recurrence from radiation necrosis, and delineate the tumor volume because of infiltrative characteristics, which could affect the treatment outcomes related with PFS and OS. However despite these limitations, our study showed that radiosurgery had a survival benefit for patients with recurrent high-grade gliomas. 


\section{CONCLUSION}

GKR could be an additional treatment option in recurrent high-grade glioma, particularly in patients with good PS and small tumor volume.

\section{CONFLICTS OF INTEREST}

No potential conflict of interest relevant to this article was reported.

\section{INFORMED CONSENT}

This type of study does not require informed consent.

\section{- Acknowledgements}

This study was supported by the Basic Science Research Program through the National Research Foundation of Korea (NRF), funded by the Ministry of Science, ICT, \& Future Planning (2017R1A1A1A05001020).

\section{References}

1. Bokstein F, Blumenthal DT, Corn BW, Gez E, Matceyevsky D, Shtraus N, et al. : Stereotactic radiosurgery (SRS) in high-grade glioma: judicious selection of small target volumes improves results. J Neurooncol 126 : 551-557, 2016

2. Combs SE, Debus J, Schulz-Ertner D : Radiotherapeutic alternatives for previously irradiated recurrent gliomas. BMC Cancer 7 : 167, 2007

3. Conti A, Pontoriero A, Arpa D, Siragusa C, Tomasello C, Romanelli P, et al. : Efficacy and toxicity of CyberKnife re-irradiation and "dose dense" temozolomide for recurrent gliomas. Acta Neurochir (Wien) 154 : 203209, 2012

4. Dodoo E, Huffmann B, Peredo I, Grinaker H, Sinclair G, Machinis T, et al. : Increased survival using delayed gamma knife radiosurgery for recurrent high-grade glioma: a feasibility study. World Neurosurg 82 : e623-e632, 2014

5. Dong Y, Fu C, Guan H, Zhang T, Zhang Z, Zhou T, et al. : Re-irradiation al- ternatives for recurrent high-grade glioma. Oncol Lett 12 : 2261-2270, 2016

6. Ernst-Stecken A, Ganslandt O, Lambrecht U, Sauer R, Grabenbauer G : Survival and quality of life after hypofractionated stereotactic radiotherapy for recurrent malignant glioma. J Neurooncol 81 : 287-294, 2007

7. Fogh S, Glass C, Andrews DW, Werner-Wasik M : Multiple courses of stereotactic re-irradiation in recurrent oligodendroglioma: a case report. J Med Case Rep 5 : 183, 2011

8. Kano H, Niranjan A, Khan A, Flickinger JC, Kondziolka D, Lieberman F, et al. : Does radiosurgery have a role in the management of oligodendrogliomas? J Neurosurg 110 : 564-571, 2009

9. Kim HR, Kim KH, Kong DS, Seol HJ, Nam DH, Lim DH, et al. : Outcome of salvage treatment for recurrent glioblastoma. J Clin Neurosci 22 : 468-473, 2015

10. Kong DS, Lee JI, Park K, Kim JH, Lim DH, Nam DH : Efficacy of stereotactic radiosurgery as a salvage treatment for recurrent malignant gliomas. Cancer 112 : 2046-2051, 2008

11. Larson DA, Prados M, Lamborn KR, Smith V, Sneed PK, Chang S, et al. : Phase II study of high central dose gamma knife radiosurgery and marimastat in patients with recurrent malignant glioma. Int J Radiat Oncol Biol Phys 54 : 1397-1404, 2002

12. Minniti G, Armosini V, Salvati M, Lanzetta G, Caporello P, Mei M, et al. : Fractionated stereotactic reirradiation and concurrent temozolomide in patients with recurrent glioblastoma. J Neurooncol 103 : 683-691, 2011

13. Niyazi M, Siefert A, Schwarz SB, Ganswindt U, Kreth FW, Tonn JC, et al. : Therapeutic options for recurrent malignant glioma. Radiother Oncol $98: 1-14,2011$

14. Oken MM, Creech RH, Tormey DC, Horton J, Davis TE, McFadden ET, et al. : Toxicity and response criteria of the Eastern Cooperative Oncology Group. Am J Clin Oncol 5 : 649-655, 1982

15. Reifenberger GKJBP, Louis DN, Collins VP : Astrocytic and oligodendroglioma in Kleihues $P$ in Cavenee WK (ed) : World health organization classification of tumours, Pathology and genetics of tumours of the nervous system. IARC Press : Lyon, 2000, pp56-67

16. Sarkar A, Pollock BE, Brown PD, Gorman DA : Evaluation of gamma knife radiosurgery in the treatment of oligodendrogliomas and mixed oligodendroastrocytomas. J Neurosurg 97(5 Suppl) : 653-656, 2002

17. Sheehan JP, Lee CC : Stereotactic radiosurgery for recurrent high-grade gliomas. World Neurosurg 82 : e593-e595, 2014

18. Simonetti G, Gaviani P, Botturi A, Innocenti A, Lamperti E, Silvani A : Clinical management of grade III oligodendroglioma. Cancer Manag Res 7 : 213-223, 2015

19. Stupp R, Mason WP, van den Bent MJ, Weller M, Fisher B, Taphoorn MJ, et al. : Radiotherapy plus concomitant and adjuvant temozolomide for glioblastoma. N Engl J Med 352 : 987-996, 2005 